\title{
Entropy, semantic relatedness and proximity
}

\author{
Lance W. Hahn • Robert M. Sivley
}

Published online: 10 May 2011

(C) Psychonomic Society, Inc. 2011

\begin{abstract}
Although word co-occurrences within a document have been demonstrated to be semantically useful, word interactions over a local range have been largely neglected by psychologists due to practical challenges. Shannon's (Bell Systems Technical Journal, 27, 379-423, 623-665, 1948) conceptualization of information theory suggests that these interactions should be useful for understanding communication. Computational advances make an examination of local word-word interactions possible for a large text corpus. We used Brants and Franz's (2006) dataset to generate conditional probabilities for 62,474 word pairs and entropy calculations for 9,917 words in Nelson, McEvoy, and Schreiber's (Behavior Research Methods, Instruments, \& Computers, $36,402-407,2004)$ free association norms. Semantic associativity correlated moderately with the probabilities and was stronger when the two words were not adjacent. The number of semantic associates for a word and the entropy of a word were also correlated. Finally, language entropy decreases from 11 bits for single words to 6 bits per word for four-word sequences. The probabilities and entropies discussed here are included in the supplemental materials for the article.
\end{abstract}

Keywords Information theory - Text corpus - Semantic association · Entropy $\cdot$ Word prediction

Electronic supplementary material The online version of this article (doi:10.3758/s13428-011-0087-7) contains supplementary material, which is available to authorized users.

L. W. Hahn $(\bowtie)$

Department of Psychology,

Western Kentucky University,

1906 College Heights Blvd. \#21030,

Bowling Green, KY 42101, USA

e-mail: Lance.Hahn@WKU.edu

\section{R. M. Sivley}

Department of Mathematics and Computer Science,

Western Kentucky University,

1906 College Heights Blvd. \#21030,

Bowling Green, KY 42101, USA
An experienced reader quickly acquires the meaning of text by efficiently recognizing words and the meanings associated with the words. In turn, the meaning of a word can accelerate subsequent word recognition, as has been repeatedly demonstrated with semantic priming (McNamara, 2005). Although word recognition integrates different sources of information, some of this acceleration is due to semantic features that are shared by the previous words and the word currently being read (Hutchison, Balota, Cortese, \& Watson 2008).

While semantic priming effects are typically described as interactions between a prime word and a target word, the broader context of a sentence also plays a role in comprehension. Many event-related potential (ERP) studies over the past decades have described how words early in a sentence create a context and an expectation for relevant words late in a sentence (e.g., Federmeier, Wlotko, De Ochoa-Dewald, \& Kutas, 2007; Kutas \& Hillyard, 1984). Such expectations can include grammatical attributes of the word, such as gender agreement (Wicha, Moreno, \& Kutas, 2004), or even a specific word in text or spoken language (Van Berkum, Brown, Zwitserlood, Kooijman, \& Hagoort, 2005).

Expectation effects produced by context also influence the time a reader fixates on a target word. The context of a sentence can reduce a word's fixation duration when the context matches the word and provides some general constraint (Balota, Pollatsek, \& Rayner, 1985; Ehrlich \& Rayner, 1981; Rayner, Ashby, Pollatsek, \& Reichle, 2004; Rayner \& Well, 1996). McDonald and Shillcock (2003) have suggested that this contextual constraint may be largely due to the relationship between the noun and verb contained in the sentence. They described the noun-verb association in terms of an objective transitional probability derived from a text corpus rather than an empirically determined expectation. Although there is some debate about the nature of the mechanisms underlying the changes in fixation duration (Frisson, Rayner, \& Pickering, 2005; Schustack, Ehrlich, \& 
Rayner, 1987), it is clear that the initial sentence context influences fixation duration. Thus, semantic similarity and contextual expectation both play important roles in efficient word comprehension, as observed in ERP and eye movement data. The main goal of this article is to determine the extent to which semantic similarity and expectancy are related within the local context of a few words.

Separating semantic similarity and predictability is a nontrivial and, in some cases, undesirable undertaking. For instance, latent semantic analysis (LSA) is a powerful way to infer the semantic similarity of words or documents that is derived from the co-occurrence of words across many documents (Landauer \& Dumais, 1997). In essence, LSA infers semantic relationships from co-occurrence statistics. Griffiths, Steyvers and Tenenbaum (2007) have taken an approach similar to LSA, with the goal of automatically creating or identifying topic models associated with a document. Each model represents a topic as a set of probabilities that specific words will be produced in text representing the topic. Again, the probability that a word is present in a document is used to infer the meaning of the text or to link text with a topic.

It is reasonable and useful to expect semantically related words to appear together in a document. However, the goal of LSA and topic models is not really to describe sentence comprehension, but to create a model of how words are linked to specific topics or documents and how words that have similar links are likely to be semantically related. These models may be capturing important aspects of discourse processing, but they are also missing important elements of reading. For instance, both of these models use a bag-ofwords approach in which grammatical rules and word order information are discarded. As a result, these approaches can predict that a document that includes the word DOCTOR might be reasonably expected to also include the word NURSE, but they cannot predict that these two words are unlikely to be immediate neighbors or likely to be in the same phrase.

Lund and Burgess (1996) have also proposed a statisticsbased model of semantic memory named HAL that limits the co-occurrence context to a 10 -word frame. By limiting the contributing co-occurrences to 10 words, words that are not in the same sentence are less likely to form associations. They allow proximity to further influence their representation by having the co-occurrence weighted as inversely proportional to the distance between the co-occurring words within the frame. In other words, the closer two words are within the frame, the stronger the weight given to that cooccurrence. HAL and variants of it have been used to model a range of behavioral results (Huettig, Quinlan, McDonald \& Altmann, 2006; McDonald, 2000; McDonald \& Lowe, 1998).

While the semantics of a word and the expected presence of a word are entangled within the context of a document, neurophysiological evidence suggests that semantics and expectation may be separable but collaborating reading processes. DeLong, Urbach and Kutas (2005) measured ERPs to determine the impact of expectancy on indefinite articles when a subsequent noun is expected. Even though indefinite articles are very limited in semantic content, they appear to be influenced by expectancy and also to provide contextual constraint. In other words, they have a role in prediction even without much semantic value.

Independent of the hypothesis that semantics and predictability may be physiologically separable, word anticipation is being recognized as an important process in reading. Kutas and colleagues have repeatedly demonstrated the impact of sentence context on ERP recordings of active word recognition (DeLong et al., 2005; Federmeier \& Kutas, 1999; Kutas \& Federmeier, 2011; Kutas \& Hillyard, 1984). The amplitude of the N400 produced by a word ending a sentence is strongly correlated with the expectancy associated with the word. This expectancy has been measured using a cloze procedure to empirically determine the probability that the word will end the sentence. Expectations generated by a sentence influence word recognition, as measured physiologically.

Expectations have also been proposed as part of a computational model to explain the effects of word frequency and the properties of a word's orthographic neighborhood on lexical decision tasks (Norris, 2006, 2009). Norris's "Bayesian Reader" uses a Bayesian decision rule to produce the "word" or "nonword" response based on the similarity of the letter input to real words and the frequency of the real words. The success of the Bayesian Reader suggests that word frequency is also a component of the predictability that influences reading.

\section{Word prediction}

While word frequency has influenced our understanding of human language comprehension since Kučera and Francis (1967) provided a large list of English word frequencies, the frequency of word sequences ( $n$-grams) has had limited influence on the psychological view of semantics in reading. In spite of this limited influence in psychology, $n$-grams have had a profound influence on computational approaches to language, including automatic speech recognition (Jelinek, 1976), machine translation (Brown et al., 1990) and other aspects of natural language processing (Berger, Della Pietra, \& Della Pietra, 1996). Shannon (1948) was the first to acknowledge the potential value of both word frequencies and word sequence probabilities in helping to understand language, in his seminal article outlining a mathematical approach to communication. Shannon demonstrated that stochastic processes that incor- 
porate information sources such as letter probabilities, letter sequence probabilities, word probabilities, and word sequence probabilities become better estimates of English text with each additional information source. Shannon depended largely on word frequencies, but he did use some two-word sequence information in at least one demonstration. He lamented (Shannon, 1948) that word sequences longer than two words would be interesting to consider but, given the limited technology available to him, "the labor involved becomes enormous at the next stage" (p. 8). One aim of this article is to use current technology to answer questions about language entropy and word entropy that were not feasible for Shannon to approach.

One potential way to extend probabilities beyond independent word frequencies to two-word sequences is to utilize the free association task. This is not the two-word sequence information source that Shannon sought, but it is a way to assess the potential link between a pair of words. Free association in response to a cue word has been used to assess the impact of a single-word context on a second word's accessibility in semantic memory (Nelson, McEvoy, \& Dennis, 2000). The extent to which free association data are related to co-occurrence frequencies is an empirical question addressed later in this report. Just as the influence of word frequency on our understanding of language has benefited from Kučera and Francis's (1967) database, the influence of free association has benefited from a large dataset in the form of Nelson et al. (2004) free association norms (FA norms). The dataset describes the associative links between 72,000 word pairs derived from a singleresponse free association task.

There have also been links generated between free association probabilities and the probabilistic relationships between specific words and a topic. Griffiths, et al. (2007) topic model is based on the assumption that a document describing a specific topic will likely include a set of words associated with the specific topic. They directly measured the probability of a word occurring for a specific topic in a large text corpus and then inferred free association rank based on the probability of one word occurring, given the probability of the other word. The result was a prediction of free association behavior that outperformed LSA.

Probability or expectation has also influenced our understanding of sentence comprehension in the form of empirically determined expectations rather than corpus-based probabilities. Determining the probability that an arbitrary sentence ends with a specific word is still not computationally feasible, but a reasonable approximation can be determined with the cloze procedure (Kutas \& Hillyard, 1984). In brief, the cloze procedure has participants complete an incomplete target sentence with a word. The distribution of responses is the basis for a set of cloze probabilities for words generated by the participants. The cloze probabilities may be similar to the actual probability that a word will end a sentence in common usage, but with some important differences. Expectancy measured behaviorally will be shaped by behavioral factors such as the availability heuristic (Tversky \& Kahneman, 1974). Cloze probabilities are also limited by methodological constraints. For instance, some sentences may have low-probability word completions that may not arise without inordinately large sample sizes (e.g., "He looked at the slice of an array."). In order to create a cloze probability, each sentence fragment must be evaluated by many participants. As a result, generating cloze probabilities for a large collection of arbitrary sentences can be very time consuming.

Behavioral measures of word expectancy in the form of a free association task or a cloze task may provide reasonable estimates of how a word or group of words predicts the occurrence of another word. They are only estimates, however. Thus far, the influence of true word probabilities in the psychological study of reading falls primarily into one of two extreme conditions: the probability that a word will occur given no other information (word frequencies) or the probability that a word will occur within a document. To our knowledge, the probability of a specific word occurring given the local occurrence of another word in typical usage has not been described for a substantive number of words. One goal in this study is to generate a set of probabilities that describes the predictive influence of one word on the expectation of another associated word within a local context.

Although word prediction based on $n$-gram statistics has yet to play a substantial role in psychology, the approach has been used to create word prediction systems for people with disabilities. Initially, word prediction systems were based on the initial characters of a word (Hunnicutt, 1985), but these systems have been expanded to include $n$-gram frequency and semantic information (Carlberger et al., 1997). The semantic information is typically introduced as categorical tags (e.g., inanimate) for words (Carlberger et al., 1997) or derived from computational strategies such as LSA (Wandmacher \& Antoine, 2007). Although LSA has influenced psychological approaches to word comprehension, $n$-gram statistics and word prediction have had a more limited impact on our understanding of human word comprehension.

In order to generate the probabilities describing the influence of local context, we need a very large text corpus or a very large record of local word contexts. Brants and Franz (2006) have generated a very large list of word sequences ( $n$-grams) and their frequencies for word sequences ranging from one to five words. These $n$-gram frequencies provide a measure that is truly analogous to traditional word frequency datasets. The frequencies were 
calculated from over 95 billion sentences available to Google through public Web pages in January of 2006. We will refer to this dataset as the Google dataset. These $n$ gram frequencies can be used as the basis for probability calculations such as the probability of a specific word, the probability of a specific $n$-gram, or the probability of one word conditioned on the presence of another word. The probability of a word occurring in an $n$-gram given that the $n$-gram begins with a specific word is the type of multipleword information that Shannon desired but was unable to generate. For instance, knowing the probability of encountering the word NURSE when DOCTOR has just been encountered provides a real measure of whether DOCTOR predicts NURSE. The Google dataset provides this type of information within a window of up to five words. The first objective of this article is the creation of a dataset that describes the predictive relationship for a large set of word pairs.

The second objective of this article is to determine whether or not relative word position is useful in describing the relationship between two words. A question that arises when considering the window of text provided by the $n$ gram is whether or not the position of a word within the window is an important factor. On one hand, the $n$-gram could be evaluated using a bag-of-words strategy in which neither order nor distance between words is considered. This strategy is commonly used when calculating the probability of a word occurring within a document. On the other hand, the relative distance between two words may be a useful source of information. For instance, content words may be immediately followed by high-frequency function words that are neither well predicted nor very useful in predicting subsequent words. We hypothesize that related words will be separated by at least one word.

A third objective of this article is to measure entropy for the English language using word sequences ranging from one to five words and to measure the entropy associated with $n$-grams beginning with a specific word. The high frequency of function words such as THE and OF make it unlikely that these words will be useful when considering the prediction or expectation of another word. An $n$-gram that begins with a function word will be highly unconstrained in what the subsequent words will be. Shannon (1948) proposed conditional entropy as a measure of uncertainty given that an event has occurred, such as a word beginning an $n$-gram, and entropy as a measure of how much uncertainty a language generally has. Although Shannon focused primarily on the probability of letters, he did estimate the entropy of English messages based on word frequencies as 11.82 bits per word (Shannon, 1951).

To summarize, this study has three objectives. First, we will create and describe a dataset that captures the predictive relationship between the large set of word pairs contained in Nelson et al.'s (2004) FA norms. Second, we will evaluate the role of word position within an $n$-gram in the predictive relationship between two words. Third, word entropy will be calculated for the English language with each set of sequences (single-word through five-gram), and conditional entropy will be calculated for $n$-grams beginning with the words from the FA-norm dataset.

\section{Experiment 1}

Our first objective was to determine the extent to which one word predicts the presence of another. Considering the relationships between all arbitrary word pairings is not feasible and would result in an overwhelming number of null results without predictive value. In order to narrow the focus to word pairs that might be reasonably expected to have a predictive relationship, we evaluated all of the word pairs in Nelson et al.'s (2004) FA norms. The predictive relationship was calculated for each word pair with (1) the cue word $(Q)$ preceding the response word $(R)$ and (2) the response word preceding the cue word. The two probabilities describing a word pair $(Q, R)$ are the probability that the response word occurs at least once following an initial instance of the cue word, $p\left(w_{2,3,4}=R \mid w_{1}=Q\right)$ and the probability that the cue word occurs at least once following an initial instance of the response word, $p\left(w_{2,3,4}=Q \mid w_{1}=R\right)$. In the probability notations, the position of the word within the $n$-gram is indicated with a subscript. A sequence of subscripts indicates a range of word positions in which the target is expected to occur at least once.

\section{Method}

Materials Brants and Franz's (2006) Web 1T 5-gram Version 1 database was acquired from the Linguistic Data Consortium and is the basis for all of the frequency and cooccurrence calculations. For complete details about the dataset see Brants and Franz (2006), but some relevant details are included here. The source data for Brants and Franz's frequency calculations were the English-language public Web pages available to Google in January 2006. Text was parsed into lists of tokens (e.g., words) by separating text at spaces or punctuation marks. This parsing created over 1 trillion tokens. The frequency datasets include tokens occurring at least 200 times and $n$-grams occurring at least 40 times. The frequency data are grouped by sequence length from one token (13.6 million unique entries) to five tokens ( 1.18 billion unique entries). The largest number of unique $n$-grams is 1.31 billion unique entries, for the four-gram frequency dataset. It is not clear why the number of unique $n$-grams peaks for a sequence 
length of four rather than five words, but one likely possibility is that extending the sequence to five words creates a sequence divergence that reduces a substantial number of $n$-gram frequencies to below 40 . If a four-gram entry had a frequency of 45 (e.g., A B C D 45) it would be included in the four-gram dataset, but extending the sequence in the five-gram dataset might produce more than one unique five-gram with frequencies less than 40 (e.g., A B C D E 25; A B C D F 20). These new five-grams would be excluded from the dataset due to their low frequencies. We will refer to this problem as the divergence problem. The word-pair analyses were conducted on the four-gram frequency data.

Compound words such as AIR FORCE were excluded from our analysis, because they were treated as two tokens in Brants and Franz's (2006) analysis but as single words in the FA norms. Word pairs that included numbers (SPY, 007) or other nonalphabetic characters were also excluded from the analysis. A total of 62,474 word pairs were analyzed.

Procedure Each unique $n$-gram sequence is listed in Brants and Franz's (2006) dataset with the frequency associated with the $n$-gram. For an $n$-gram with four words $\left(\begin{array}{llll}w_{1} & w_{2} & w_{3} & w_{4}\end{array}\right)$, the probability was calculated that the response word $(R)$ occurred in the $n$-gram, given that the cue word $(Q)$ was in the first position, and we will represent the calculation as:

$$
\begin{aligned}
& p\left(w_{2,3,4}=R \mid w_{1}=Q\right) \\
& \quad=\frac{\Sigma C\left(Q R w_{3} w_{4}\right)+\Sigma C\left(Q w_{2} R w_{4}\right)+\Sigma C\left(Q w_{2} w_{3} R\right)-D}{\Sigma C\left(Q w_{2} w_{3} w_{4}\right)}
\end{aligned}
$$

$D=\Sigma C\left(Q R R w_{4} \vee Q w_{2} R R \vee Q R w_{3} R\right)+\Sigma C(Q R R R)$

$C\left(\begin{array}{llll}w_{1} & w_{2} & w_{3} & \left.w_{4}\right)\end{array}\right)$ represents the number of times the $\left(w_{1}\right.$ $\left.w_{2} w_{3} w_{4}\right) n$-gram occurred in the corpus. For each quantity, the summation is across all $n$-gram patterns that match the word sequence indicated within the summation. For instance, $\sum C\left(Q R w_{3} w_{4}\right)$ represents the sum of counts for all $n$-grams that begin with the cue word and response word followed by any subsequent pair of words. The definition of $D$ in Equation 2 accumulates the number of times an $n$ gram with multiple instances of $R$ occurs. In Equation 2, any $n$-gram with exactly two instances of $R$ is summed in the first summation, and any $n$-gram with exactly three instances of $R$ is summed in the second summation. Subtracting by $D$ removes the duplicate counts produced when $R$ is present more than once in the $n$-gram, which ensures that each $n$-gram in the corpus is counted only once. In this calculation, the $n$-gram "flew up up up" would contribute to $\sum C\left(Q R w_{3} w_{4}\right), \sum C\left(Q w_{2} R w_{4}\right)$, and $\sum C$ $\left(Q w_{2} w_{3} R\right)$, but two of these instances would be removed by subtracting $D$, which counts this $n$-gram twice. One benefit of the conditional probability calculation in Equation 1 is that it utilizes separate sums targeting $R$ in specific positions. In Experiment 2, the value of the position-specific information provided by these sums is considered.

Since the cue precedes the response, we consider $p\left(w_{2,3,4}=\right.$ $R \mid w_{1}=Q$ ) to be analogous to the forward strength measure reported in the FA norms. The ordering of the cue and response was reversed to calculate $p\left(w_{2}, 3,4=Q \mid W_{1}=R\right)$, which was analogous to the backward strength measure reported in the FA norms.

The analysis program was written in Java and ran for several days on a collection of Windows-based PCs. The total computation time for both Experiments 1 and 2 was approximately 160 days (if a single computer had been used). For each lowercase cue word, a file (or files) in the Google dataset was selected using the index files provided with the Google dataset. The program scanned the identified file(s) for word sequences that began with the cue word and contained the response word in one of the subsequent positions. The case of the letters (upper or lower) was ignored during the scanning process. In order to provide a complete set of word-pair statistics for links from the response back to the cue, the analysis program was also run with the cue and response words in reverse order. These links from the response to the cue were not important for the present question of whether word prediction is related to forward association strength, but the links could be important for future computational models of semantic and reading processes.

\section{Results and discussion}

The ordered co-occurrence frequencies and probabilities are included in the supplemental materials. The correlation between FA norm forward strengths $(M=.032, S D=.076)$ and $p\left(w_{2,3,4}=R \mid w_{1}=Q\right)(M=.005, S D=.025)$ was modest but significant $[r=.151, \mathrm{t}=38.22, \mathrm{df}=62472), p<.0001]$. This finding is consistent with a modest overlap between semantic associativity and predictability across the long list of words assessed.

Several factors may keep the correlations between the FA norms and predictive links low. Nelson et al. (2000) have suggested that the single-response free association task underestimates the number of associates for a given target because of its focus on the strongest associates available. Weak associates that are absent in the FA norms may be present in the $n$-gram data and dilute the correlation with FA norms.

The presence of multiple meanings (or senses) and the difficulty in determining which meaning is being used has been 
acknowledged as a very difficult computational problem (Navigli, 2009). While some divergent word senses do occur in the FA norms (e.g., LABOR produces WORK and PREGNANT), other divergent meanings do not (e.g., BANK produces MONEY but not RIVER). To the extent to which these multiple meanings are mutually exclusive in an $n$-gram, they compete and dilute the relationship between the FA norms and the predictive links. In other words, by treating a word as if it has only one meaning, the correlation between FA norms and predictive links is reduced. The magnitude of this reduction will vary with the number and usage characteristics of the different meanings. Future research may be able to use the entropy associated with a word (see Exp. 4) to estimate the impact of multiple meanings on a specific word by assuming that a word with multiple meanings is likely to be followed by a more diverse set of words than a word with a single meaning.

When graph theory is applied to human semantic memory, there is sometimes a simplifying assumption that the direction of a link between nodes (words within the context of a semantic network) can be ignored. This occurs because undirected graphs are simpler and more mathematically approachable than directed graphs. For a brief introduction to graph theory and a relevant discussion of this topic, see Steyvers and Tenenbaum (2005). The very small correlation $(r=.033, \mathrm{t}=8.21, \mathrm{df}=62472, p<.0001)$ between $p\left(w_{2,3}\right.$, $\left.{ }_{4}=R \mid w_{1}=Q\right)$ and $p\left(w_{2,3,4}=Q \mid w_{1}=R\right)(M=.005, S D=$ .026) suggests that this simplifying assumption should be avoided for word-word predictions.

\section{Experiment 2}

In Experiment 1, the co-occurrence calculations considered the order of the cue and response words but did not consider the distance between the words within the fourword window. It is conceivable that the predictive probability may decline as the distance between the words increases (Lund \& Burgess, 1996). It is also possible that semantically related words may not typically be immediate neighbors because they may have function words between them. To consider these possibilities, probabilities were calculated for specific positions within the $n$-gram rather than simply for whether or not the word was present within the $n$-gram. As in Experiment 1, the focus was limited to words included in the FA norms.

Method

Materials The same materials from Experiment 1 were used in Experiment 2.

Procedure The procedure from Experiment 1 was used in Experiment 2, with probabilities calculated for each specific location within the four-gram with either the cue or the response word in the first $n$-gram position. These probabilities are $p\left(w_{2}=R \mid w_{1}=Q\right), p\left(w_{3}=R \mid w_{1}=Q\right), p$ $\left(w_{4}=R \mid w_{1}=Q\right), p\left(w_{2}=Q \mid w_{1}=R\right), p\left(w_{3}=Q \mid w_{1}=\right.$ $R)$, and $p\left(w_{4}=Q \mid w_{1}=R\right)$.

\section{Results and discussion}

The correlations between forward association strength and the three position-specific probabilities are shown in Table 1. As hypothesized, the weakest association was between the forward strength and the probability of the associate being the immediate neighbor. The probability of the associate being in Positions 3 and 4 generated probabilities that rivaled the position-independent probabilities measured in Experiment 1. This result suggests that word position is important and that semantically related words are not typically immediate neighbors.

These correlations indicate that weighting cooccurrences according to an inverse proportion (Lund \& Burgess, 1996) may not be appropriate for four-word $n$ grams. An inversely weighted frequency was calculated for each word and is included in the supplemental materials. Although the unweighted frequency was slightly more correlated $(r=.0087)$ with forward strength than was the weighted frequency $(r=.0072)$, both correlations were small and very similar. Within this local context, a weighting scheme may have a limited impact.

\section{Experiment 3}

Shannon (1948) founded the information theory approach in psychology with his mathematical description of the inherent predictability or entropy within the English language. By knowing the predictability of the signal (e.g., English text), we gain an understanding of the bandwidth required for a channel or link to accommodate the signal. Entropy provides an overall measure of a language's predictability and, in turn, tells us how flexible

Table 1 Correlations as a function of associate word position

\begin{tabular}{lll}
\hline Word Position Probability & Correlation with USF FSG & $t$ \\
\hline$p\left(w_{2}=R \mid w_{1}=Q\right)$ & .064 & $16.05^{*}$ \\
$p\left(w_{3}=R \mid w_{1}=Q\right)$ & .168 & $42.73^{*}$ \\
$p\left(w_{4}=R \mid w_{1}=Q\right)$ & .131 & $33.13^{*}$ \\
$p\left(w_{2,3,4}=R \mid w_{1}=Q\right)$ & .151 & $38.23^{*}$ \\
\hline
\end{tabular}

USF FSG, University of South Florida free association norm forward strength. ${ }^{*} p<.0001$. 
a model of language must be in order to accommodate the language.

Entropy has also been the focus of a recently observed type of constancy in language. Genzel and Charniak (2002) proposed that typical communication is influenced by a "constancy rate principle" that increases local measures of entropy at a constant rate as communication progresses. For example, as a reader gains experience with a document by reading text, the writer increases the entropy of the text on a local scale. The theoretical goal of the increased local entropy is to maximally use the available bandwidth of the communication channel. The trend of increasing local entropy has been described using Wall Street Journal articles (Genzel \& Charniak, 2002), within paragraphs and across languages (Genzel \& Charniak, 2003), and in telephone conversations (Frank \& Jaeger, 2008; Jaeger, 2010; Levy \& Jaeger, 2007). The speaker can alter local entropy by making choices about contraction usage (Frank \& Jaeger, 2008) and using optional words such as "that" (Jaeger, 2010). Estimating the maximum capacity of the communication channel in terms of entropy is the primary focus of this study.

Before measuring the entropy of a system, it is necessary to establish the level of encoding used within the system. Previous entropy measures of English have often focused on the letter level of encoding because communication devices often operate at this level and because measuring entropy at the word level of encoding is technically challenging, due to the size of the dataset. At the letter level of encoding, English appears to have an entropy of between 1.3 (Shannon, 1951) and 1.75 (Brown, Della Pietra, Della Pietra, Lai, \& Mercer, 1992) bits per character. Shannon's (1951) measure was generated behaviorally by asking participants to guess which letter would be next in a sequence of letters taken from written text, while Brown et al. (1992) measured entropy using a corpus-based approach.

In addition to establishing the level of encoding, it is necessary to decide the size of each sample. For instance, the distribution of word frequencies can be used to describe language entropy, but this ignores the relationships between words that allow us to recognize "vice president" as common but "vice stapler" as uncommon. Based on word frequencies, Shannon (1951) estimated word entropy for English as 11.82 bits per word, but there has been some debate about this estimate, with Grignetti (1964) estimating entropy to be 9.8 bits per word. Gathering frequencies for phrases composed of two or more words is technically challenging, but Brants and Franz (2006) created such frequencies for all word sequences up to five words in length that occurred at least 40 times in the Google dataset. Our objective in this study was to measure the entropy of English at the word level for $n$-gram lengths ranging from one to five words. These results will measure the inherent variability of English as well as inform us about the sequence length that is useful in language processing.

\section{Method}

Materials The basis for these analyses was the Google dataset files that provided $n$-gram frequencies for sequences that began with a lowercase letter. The range of $n$-gram entries was limited to those that were in the inclusive range beginning with "a" and ending with "zzzz" as the first word in the $n$-gram sequence.

Procedure Shannon (1948) provided the equation for entropy as

Entropy $=-\sum_{i=1}^{k}\left(p\left(w_{i}\right) \times \log \left(p\left(w_{i}\right)\right)\right)$

This equation assumes that $n$-grams $\left(w_{i}\right)$ are independent of one another. While this is true for single words, it is not true for longer sequences, because the sequences overlap. For instance, the sequence (A B C) would contribute to both bigrams (A B) and (B C), with $\mathrm{B}$ in the overlapping region. Using this equation for a word sequence rather than a word is calculating joint entropy, which is not a true measure of the entropy of an English word. Shannon (1951) proposed that language entropy be measured as the limit of $n$-gram entropy (Eq. 4) as the $n$-gram length approaches infinity. In Equation $4, w_{i}^{N}$ represents the $i$ th $n$-gram of length $N$.

$$
\begin{aligned}
\text { Entropy }_{N}= & -\sum_{i=1}^{k}\left(p\left(w_{i}^{N}\right) \times \log \left(p\left(w_{i}^{N}\right)\right)\right) \\
& +\sum_{i=1}^{k}\left(p\left(w_{i}^{N-1}\right) \times \log \left(p\left(w_{i}^{N-1}\right)\right)\right)
\end{aligned}
$$

Calculating the exact probability that a particular $n$-gram will occur is very difficult, because only estimations of common usage are possible. Although the Google dataset is quite large, it is not a complete description of all possible English usage. Furthermore, Brants and Franz's (2006) requirement that all $n$-grams occur at least 40 times in order to be included means that low-frequency $n$-grams are absent. Computational linguists have developed a variety of methods (Chen \& Goodman, 1998; Chen \& Rosenfeld, 1999; Good, 1953; Talbot \& Osborne, 2007; Zhai \& Lafferty, 2004) to reduce the impact of the missing $n$-gram counts. The methods, called smoothing methods, attempt to remove the influence of sparse data by smoothing the probability distribution. While an exploration of smoothing methods is beyond the scope of this work, a simple 
smoothing approach is provided to indicate the impact smoothing might have on the entropy measured.

We adopted a back-off strategy (Chen \& Goodman, 1998 ) to address the problem of the missing $n$-gram counts. We began the adjustment process by adopting Brants and Franz (2006) assumption that all useful words occur on the Internet at least 200 times and, as a result, the single-word entropy is unadjusted. However, requiring an $n$-gram to occur at least 40 times has the potential of creating a measurable effect, especially on the relatively long fiveword $n$-grams. We estimated the total number of observed $n$-grams lost when the sequence length is increased from $n$ to a length of $n+1$ as the difference between the totaled frequencies. For instance, 4 million unique single words were observed and occurred a total of 371 billion times, but the 110 million unique bigrams occurred only 365 billion times. Thus, we estimate the number of lost bigrams as 6 billion (the difference between the number of single words and the number of bigrams). Some of these bigrams will be excluded because they occur at least once but fewer than 40 times. To estimate the largest impact of these excluded $n$ grams, we adopt the extreme position of assuming that all lost $n$-grams occur only once. Admittedly, this still does not take into account the $n$-grams that are possible but absent from the corpus, but these unobserved $n$-grams are likely to have limited psychological relevance. We applied this backoff strategy recursively so that, for example, the adjusted trigram entropy calculation estimates the excluded trigrams as the sum of the estimated bigrams excluded and the estimated trigrams excluded.

Two computer programs written in the programming language Perl were used to calculate the sum of the frequency $\mathrm{x} \log$ (frequency) products for each file in the Google dataset and the total sum of the frequencies for each file.

\section{Results and discussion}

The estimates of English entropy based on $n$-grams ranging from one to five words with and without the adjustment are plotted in Fig. 1. The entropy for single words (10.94 bits) was similar to Shannon's (1951) original calculation (11.82 bits). The absence of an asymptote for the unadjusted estimates suggests that the simplifying measures taken by Brants and Franz (2006) that made data acquisition feasible have adversely affected our ability to definitively measure language entropy. However, the asymptotic appearance of the adjusted estimates for $n$-gram lengths up to 4 words is reasonably consistent with entropy estimates of 7.5 to 9 bits by Keller (2004) for English trigrams and estimates of 6.8 to 8 bits by Genzel and Charniak (2002). The adjusted entropy estimate based on five-word $n$-grams is not consistent with the other adjusted estimates and suggests

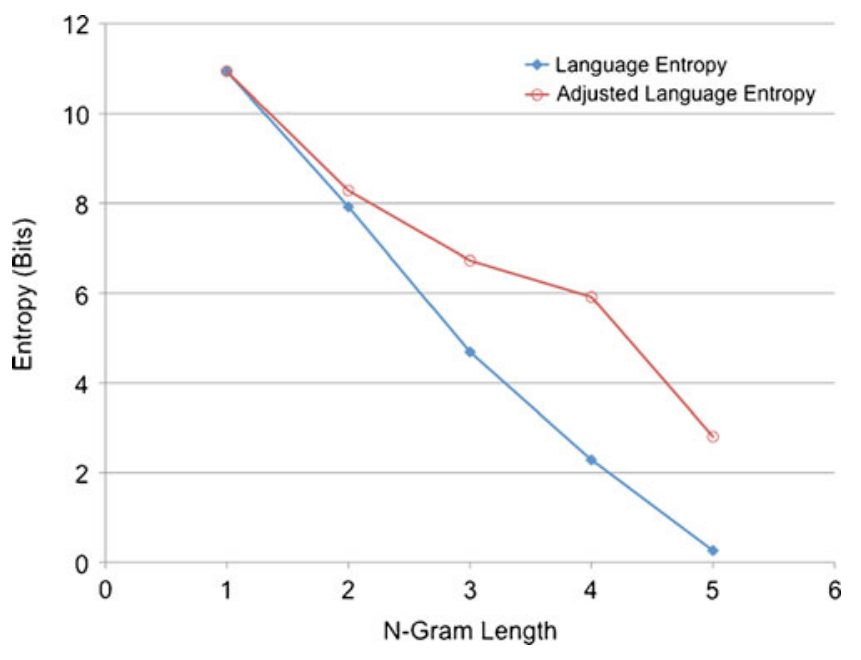

Fig. 1 Language entropy estimates based on the range of $n$-gram sizes

that the adjustment may have been overwhelmed by the loss of a substantial number of $n$-grams.

\section{Experiment 4}

While the entropy measure for a language provides a sense of the intrinsic predictability in a language and insight into the span of text that is most likely to be analytically useful, it is not particularly useful when modeling cognitive processes at the word level. One important characteristic of a word is the extent to which it influences other words. Collins and Loftus's (1975) spreading-activation model explicitly made this point by having words connect to all semantically related words. For making predictions, a word may highly constrain the following local context (e.g., "loaf") and be strongly connected to a few words, or it may weakly constrain the local context (e.g., "the") and be weakly connected to many words. One way to capture a word's ability to constrain the subsequent local context is to measure the entropy of $n$ grams that begin with the word. To serve this aim, conditional entropy was calculated for each word used in the word pairs of Experiments 1 and 2. The entropy was calculated as conditional on the target word beginning the $n$ gram. This conditional entropy describes the extent to which the presence of the target word at the beginning of the $n$ gram $\left(w_{1}=X\right)$ constrains the variability of the subsequent words in the $n$-gram $\left(A_{i}=w_{2} w_{3} w_{4}\right)$.

$\operatorname{Entropy}\left(A_{i} \mid w_{1}=X\right)$

$$
=-\sum_{i=1}^{k}\left(p\left(A_{i} \mid w_{1}=X\right) \times \log \left(p\left(A_{i} \mid w_{1}=X\right)\right)\right)
$$


The purpose of these calculations was two-fold. First, we intend to create a set of entropy calculations for words representing common English usage. By generating the entropy values associated with a large set of words, it becomes possible to create computational models that utilize word entropy as a model feature. For instance, function words can be defined as words with entropies higher than a specific entropy threshold. Entropy can also be used to describe the level of connectivity for a specific word. In order for a word to have high entropy, it must have access to a large set of connections.

The second purpose of these word entropy calculations is to compare prediction and semantics in terms of the breadth of a word's impact. The entropy of a word is somewhat analogous to the number of associates, or set size, produced by a cue word in a free association task. If predictability is modeled as a network that is analogous to a semantic network with strong links between words representing high predictive connections, then word entropy provides another way of comparing semantic and predictive information. If free association set size is correlated with word entropy, these analogous attributes may share a common neural representation. On the other hand, if these attributes are not correlated, this would suggest that associativity and predictability are separable concepts.

\section{Method}

Materials The materials described in Experiment 1 were also used in this study. The target words were the cue and response words from the FA norms. Compound words and text that included nonalphabetic characters were excluded from the analysis. A total of 9,917 words were analyzed.

Procedure The entropy computations made in the wholelanguage analysis in Experiment 3 were applied to each of the target words. Specifically, the collection of $n$-grams that begin with the target word were collected and the entropy of that set of $n$-grams was calculated. We calculated the conditional entropy (defined in Eq. 5) conditioned on the target word being the first element of the $n$-gram. The supplemental materials include the word entropy, an intermediate entropy calculation, and some descriptive statistics for each target word. The entropy computations were written in Java and run on Windows-based PCs.

Intermediate calculations were generated and included in the supplemental files for two reasons. First, some transparency in the calculations was desired, in order to allay concerns about overflow errors occurring with the very large numbers being manipulated. The second reason is that entropy can be calculated in different ways to compensate for the exclusion of low-frequency $n$-grams. These intermediate calculations will allow future research- ers to consider correction factors without enduring the arduous task of summing the logarithm of the frequency for each $n$-gram entry.

The intermediate value, described by Equation 6, is the sum of each $n$-gram count multiplied by the logarithm of the count. This calculation is the most computationally intensive task in the entropy calculation. The intermediate value produced according to Equation 6 can be converted to an entropy value for $n$-gram $A_{i}$ using Equation 7, with $m$ equal to the sum of all $C\left(A_{i}\right) n$-gram counts being considered, and $C\left(A_{i}\right)$ being the number of times $n$-gram $A_{i}$ occurs. The equivalence of Equations 3 and 6 is derived in Appendix A.

$I=\sum_{i=1}^{k}\left(C\left(A_{i}\right) \times \log \left(C\left(A_{i}\right)\right)\right)$

Entropy $=\log (m)-\frac{1}{m} I$

Results and discussion

As expected, function words dominated the high-entropy portion of the distribution. The words OF, AND, THE, A, TO, WITH, FOR, THAT, and IN were the only words with entropies greater than 19 bits. The distribution of conditional entropy is provided in Fig. 2.

The entropy associated with a target word correlated $(r=.195, t=14.04, d f=4984, p<.0001)$ with the free association set size $(M=14.38, S D=5.15)$ of the word. Some difference was expected for the two attributes, because entropy provides not only a measure of how many words are associated with the target word but also how evenly distributed probabilities are across the associated words. For instance, a set size of three might indicate three

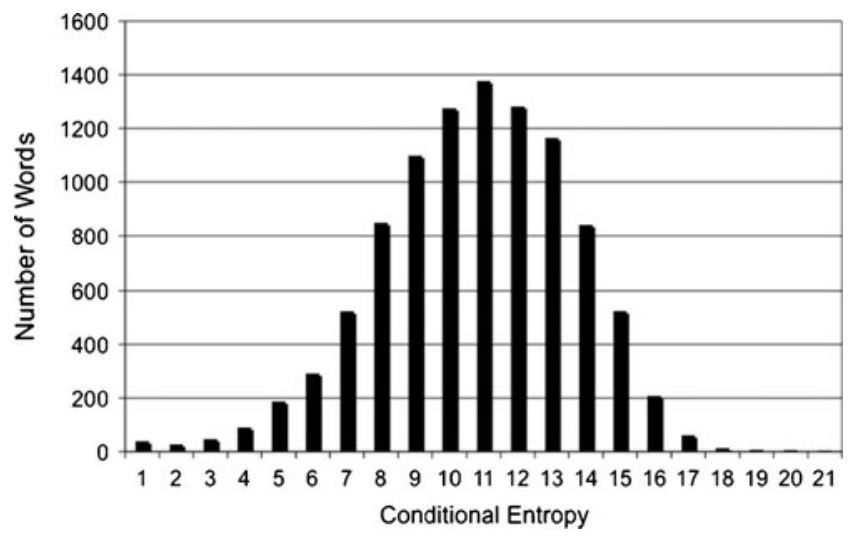

Fig. 2 The distribution of four-gram conditional word entropies for words in the University of South Florida free association norms 
equally likely associates (high entropy), or it might indicate a highly dominant associate and two very weak associates (low entropy).

The number of associates created for a target word in a free association task and the predictive value of a word appear to share some variance. The source of this shared variance cannot be inferred from this correlation study. However, Steyvers and Tenenbaum (2005) suggested that patterns of connectivity common to the FA norms, Roget's Thesaurus, and WordNet are due to common network developmental processes. The network characteristics shared by predictability and semantic set size may be due to a shared representation, but it is also quite plausible that the representations are separate despite having undergone common developmental processes.

The FA norms include part-of-speech labels for 5,052 words. Although multiple meanings for a word often make part of speech a difficult construct to limit to a specific general label (e.g., is RESEARCH a noun or a verb?), one might expect some parts of speech to be higher in entropy than others. Figure 3 shows a summary of word entropies averaged by part of speech. Note that in this analysis of the $n$-grams, we are presuming that all word usage conforms to the part of speech indicated in the FA norms. Treating RESEARCH, for example, as if it is always a noun is a gross simplification but one that is difficult to overcome (Navigli, 2009), and overcoming it is not feasible for the size of the dataset currently being examined. A general trend does occur, with more functional parts of speech, such as prepositions, generally having higher entropy than parts of speech such as nouns that are typically content words. Since the number of words in each category varies

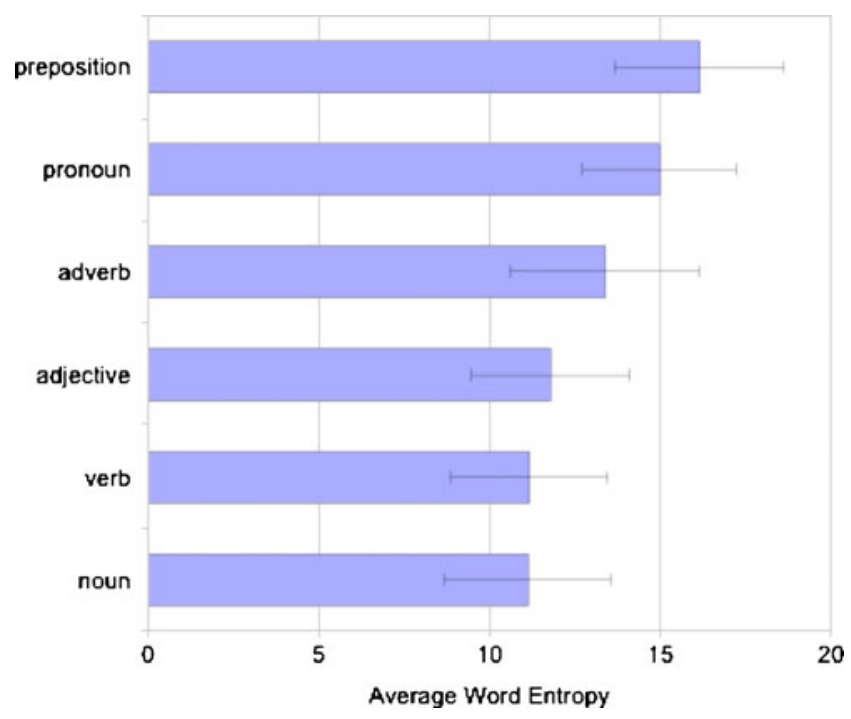

Fig. 3 The averages and standard deviations (whiskers) of word entropies for different parts of speech substantially across categories, and since there is ambiguity in the construct, the figure should be considered descriptive rather than conclusive.

Zipf's observation that word length and word frequency are inversely related (Zipf, 1935, as cited by Sigurd, EegOlofsson \& van de Weijer 2004) led us to consider the parallel relationship between word length and word entropy. We expected short words to have both high frequency and high entropy, and word entropy was indeed significantly correlated $(r=-.142, t=-14.32 d f=9915, p<.0001)$ with word length $(M=6.56$ letters, $S D=2.18)$.

\section{General discussion}

Anticipating a word from recent context

As text is being read, the experienced reader has access to many sources of information, including linguistic, contextual, and visual information. Efficient reading utilizes each of these sources to converge quickly on the recognition of a word and its associated meaning. While some contextual influences require semantic mediation (e.g., a title leads to expected text), a simple measure of local context is how well a word is predicted by the occurrence of recently viewed words. Historically, measuring this predictability has not been feasible, due to the computational labor required to measure word sequence frequencies for large text corpora.

Brants and Franz's (2006) $n$-gram frequency dataset was a substantial step toward measuring how local context foreshadows a word, but the $n$-gram frequencies alone do not capture the relationships between the words within the $n$-gram. By examining the frequencies for all $n$-grams that begin with a target word and then determining how often a second word occurs within the $n$-gram, we calculated the conditional probability $p\left(w_{2,3,4}=R \mid w_{1}=Q\right)$ describing how one word predicts the occurrence of a second word. Free association word pairs provide a natural collection of interesting word pairs with potential predictive relationships. Using these word pairs also allowed us to compare the semantic links between words with the predictive links between the words.

With the bag-of-words approach, in which the position of the word within the $n$-gram is ignored, a modest but significant correlation was found between the cue-toresponse free association strength and the cue-to-response prediction. The moderate correlation size suggests that semantic associativity and the local predictive relationship should be empirically separable, since substantial variability is exclusive to each of the two relationships. The probabilities provided in the supplemental materials should allow researchers to further examine the inde- 
pendent actions of predictive processes and semantic processes.

When the position of the word is considered in a local context, it becomes clear that the distance between the first word and the target word is important. As hypothesized, the correlation between semantic associativity and prediction strength was small when the two words were immediate neighbors and larger when the two words were separated by two or three words. This is consistent with function words acting as intermediates between semantic associates. Griffiths, Steyvers, and Tenenbaum's (2007) successful simulation of semantic associativity using predictions based on the context of a document without position information suggests that position information beyond the range of a few words is unlikely to be important to estimating semantic associativity.

Although this article has focused on simple conditional probabilities as the measure of relatedness, other metrics based on these probabilities are possible and can be derived from the supplemental materials. For example, Bullinaria and Levy (2007) evaluated and contrasted different computations for deriving word meaning from co-occurrence statistics. After comparing several computed metrics, they concluded that a normalized conditional probability called positive pointwise mutual information was the best metric. This metric can be easily calculated using the supplementary materials and does appear to produce some modest increases in the correlations between the predictive links and the association strength.

\section{Word and language entropy}

A word can highly constrain the sequence of words that immediately follow it. The word sequence following MISPLACE is constrained to include small objects that a person typically carries, such as reading glasses, keys, or a purse. On the other hand, the word sequence following THE is very unconstrained and may include a vast array of words. For the first time, our dataset of word entropies provides a measure of the constraint imposed by a word initiating a four-word sequence. The nature of the constraint may be semantic or syntactic. No matter the cause of the constraint, it is certainly predictive and is likely to be useful in normal reading.

While the predictive links between words may help anticipate specific words, the entropy of a word defines the general value of the word as a predictor. At one extreme, function words are consistently high in entropy. However, having high entropy does not mean that a word cannot have some strong predictive links. For example, one of the strongest predictive links was ABLE predicting Tо $\left(p\left(w_{2,3,4}=\right.\right.$ TO $\left.\left.\mid w_{1}=\mathrm{ABLE}\right)=.988\right)$, even though ABLE has an entropy (13.80 bits) over one standard deviation above the average and TO has an entropy (20.16 bits) over three standard deviations above the average.

On the other hand, content words generally have low entropy values that indicate a narrow set of potential sequences. However, a word with low entropy is not guaranteed to have a clear predictive link with another word. For example, MARXISM has an entropy (1.97 bits) of almost three standard deviations below average, but FASCISM (9.21 bits), which is MARXISM's only semantic associate, has a very low predictive link $\left(p\left(w_{2,3,4}=\right.\right.$ FASCISM $\mid w_{1}=$ MARXISM $)=$ .00075). Word entropy, by definition, describes the general ability of a word to predict other words, but it does not exclude the presence of strong (or weak) predictive relationships to specific words.

Expanding the focus from a word to the $n$-gram allows us to consider Shannon's (1948) original question of how much information is contained in a message. In his view, information is strictly an engineering concept that is independent of the semantic content and can be summarized as "How powerful must a communication device be in order to carry any message?" Although he foresaw the use of computers by describing information in terms of bits, his approach to communication is relevant for anything that carries communication, including neural tissue.

Shannon's (1951) calculation of English entropy as 11.82 bits was an estimation using Zipf's law, based solely on Dewey's (1929) word frequencies. Our single-word entropy (10.94 bits) was remarkably similar to Shannon's calculation, given the vastly different scales and sources of the data. Unfortunately, some assumptions and caution were necessary when inferring language entropy from the asymptotic behavior of $n$-gram entropy as the $n$-gram length increased. The unadjusted language entropy does not appear to asymptote, but instead, monotonically decreases for the measured range of $n$-gram lengths. One plausible explanation for this behavior is that the $n$-grams missing from the Google dataset due to the divergence problem distort the entropy measure so that a positive asymptote is not reached. The asymptotic behavior of the adjusted entropy as $n$-gram length approaches four words supports this explanation, but the unusual five-word adjusted entropy suggests that the five-word frequencies may be based on a substantial number of excluded $n$-grams and that these limitations is not fully overcome by the adjustment procedure.

As we would expect from the success of LSA and other co-occurrence-based approaches to semantics, word-word prediction is moderately correlated with semantic associativity. Words that share a topic are likely to co-occur in a document, and to a lesser extent, words that share meaning are likely to co-occur with one or two words separating them. Similarly, words that generate many associates in a free association task are likely to have high entropy, indicative of a diffuse prediction. 
Table 2 Attributes of Archived materials by column

\begin{tabular}{|c|c|c|}
\hline Column Number & Predictive Links Attribute & Word Entropy Attribute \\
\hline 1 & Cue Word & Cue Word \\
\hline \multirow[t]{2}{*}{2} & Response Word & Intermediate Calculation \\
\hline & & $C \log (C)$ Summed \\
\hline 3 & $C\left(Q R w_{3} w_{4}\right)$ & Conditional Entropy $\left(w_{1}=Q\right)$ \\
\hline 4 & $C\left(Q w_{2} R w_{4}\right)$ & Frequency Average \\
\hline 5 & $C\left(Q w_{2} w_{3} R\right)$ & Frequency Standard Deviation \\
\hline 6 & $C\left(w_{1}=Q\right.$ and $\left.w_{2,3,4}=R\right)$ & Highest Frequency \\
\hline 7 & $p\left(w_{2}=R \mid w_{1}=Q\right)$ & \\
\hline 8 & $p\left(w_{3}=R \mid w_{1}=Q\right)$ & \\
\hline 9 & $p\left(w_{4}=R \mid w_{1}=Q\right)$ & \\
\hline 10 & $p\left(w_{2,3,4}=R \mid w_{1}=Q\right)$ & \\
\hline 11 & Unique 4-grams with $\mathrm{Cue}_{1}$ Response $_{\mathrm{x}}$ & \\
\hline 12 & $C\left(w_{1}=Q\right)$ for 4-grams & \\
\hline 13 & $C\left(R Q w_{3} w_{4}\right)$ & \\
\hline 14 & $C\left(R w_{2} Q w_{4}\right)$ & \\
\hline 15 & $C\left(R w_{2} w_{3} Q\right)$ & \\
\hline 16 & $C\left(w_{1}=R\right.$ and $\left.w_{2,3,4}=Q\right)$ & \\
\hline 17 & $p\left(w_{2}=Q \mid w_{1}=R\right)$ & \\
\hline 18 & $p\left(w_{3}=Q \mid w_{1}=R\right)$ & \\
\hline 19 & $p\left(w_{4}=Q \mid w_{1}=R\right)$ & \\
\hline 20 & $p\left(w_{2,3,4}=Q \mid w_{1}=R\right)$ & \\
\hline 21 & Unique Four-grams with Response ${ }_{1} \mathrm{Cue}_{\mathrm{x}}$ & \\
\hline 22 & 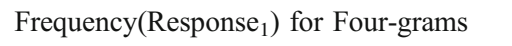 & \\
\hline 23 & Inverse Weight $\left(w_{1}=Q\right.$ and $\left.w_{2,3,4}=R\right)$ & \\
\hline 24 & Inverse Weight $\left(w_{1}=R\right.$ and $\left.w_{2,3,4}=Q\right)$ & \\
\hline
\end{tabular}

Since Shannon first described his mathematical theory of communication in 1948, scientists and engineers have used probabilities to shape communication devices. Technical advances in computation have made it possible to extend Shannon's original examination of English from letter prediction (Shannon, 1951) to predicting word sequences of up to five words. The Predictive Link and Word Entropy datasets in the supplemental materials provide a foundation in information theory that will allow psychologists to empirically and theoretically examine the influence of word-word predictions on reading processes.

Author Note This work was made possible with computational support from the College of Education and Behavioral Sciences, the Educational Technology Center, and the Department of Psychology of Western Kentucky University. We gratefully acknowledge an anonymous reviewer's assistance in clarifying the equations in this article and in improving Experiment 3.

\section{Appendix 1: Reformulation of entropy}

For several reasons, it is useful to have an intermediate calculation when calculating entropy over a very large set of events. The set of events may be distributed across several sessions or several computers. Instability in terms of computer job interruption or power interruption also makes smaller computational jobs that produce intermediate calculations desirable. There are also theoretical reasons for the present intermediate calculation. Because all $n$-grams that occur fewer than 40 times were ignored, the sparsely occurring $n$-grams are not part of the entropy calculation. For some purposes, this simplification may be undesirable.

Choosing the best corrective factor depends on the question being asked and is beyond the scope of the present paper. However, we include an intermediate value in the supplemental materials so that such corrective factors might be considered without the need to repeat the computationally intensive $p \times \log (p)$ calculation.

Entropy the entropy over a set of $k$ unique events and $m$ instances

$k \quad$ the number of unique events (the number of unique $n$-grams)

$m$ the number of instances that have occurred (the total number of $n$-grams) 
$C\left(A_{i}\right) \quad$ the number of times $n$-gram $A_{i}$ has occurred

$p\left(A_{i}\right) \quad$ the probability of unique $n$-gram $A_{i}$ occurring.

Entropy $=-\sum_{i=1}^{k}\left(p\left(A_{i}\right) \times \log \left(p\left(A_{i}\right)\right)\right)$

Entropy $=-\sum_{i=1}^{k}\left(\frac{C\left(A_{i}\right)}{m} \times \log \left(\frac{C\left(A_{i}\right)}{m}\right)\right)$

Entropy $=-\sum_{i=1}^{k}\left(\frac{C\left(A_{i}\right)}{m} \times\left[\log \left(C\left(A_{i}\right)\right)-\log (m)\right]\right)$

Entropy $=-\sum_{i=1}^{k}\left(\left[\frac{C\left(A_{i}\right)}{m} \times \log \left(C\left(A_{i}\right)\right)\right]-\left[\frac{C\left(A_{i}\right)}{m} \times \log (m)\right]\right)$

Entropy $=\frac{\log (m)}{m} \sum_{i=1}^{k}\left[C\left(A_{i}\right)\right]-\sum_{i=1}^{k}\left[\frac{C\left(A_{i}\right)}{m} \times \log \left(C\left(A_{i}\right)\right)\right]$

Since $m=\sum_{i=1}^{k} C\left(A_{i}\right)$, then

Entropy $=\log (m)-\frac{1}{m} \sum_{i=1}^{k}\left(C\left(A_{i}\right) \times \log \left(C\left(A_{i}\right)\right)\right)$

or

Entropy $=\log (m)-\frac{1}{m} I$

The reported intermediate calculation is $\sum_{i=1}^{k}\left(C\left(A_{i}\right) \times \log \right.$ $\left.\left(C\left(A_{i}\right)\right)\right)$.

\section{Archived materials}

Two main products of this work are (1) a dataset describing the predictive links between word-pairs and (2) a dataset describing the entropy characteristics for individual words. The Predictive Link dataset is organized into eight alphabetized files. The calculations for each link were generated for the directed link from the target word to the associate word (e.g., $p\left(w_{2,3,4}=R \mid w_{1}=Q\right)$ and from the associate word to the target word (e.g., $p\left(w_{2,3,4}=Q \mid w_{1}=R\right)$. Word-pairs were ordered across the files so that the first word in each pair comes alphabetically before the second word in the pair.

The Predictive Link files include 62,474 word pairs with each word pair represented as a single line of comma- delimited text. The first line in each file is descriptive text that lists the attributes in Table 2. The frequencies are simple sums of the $n$-gram frequencies that meet the word position criteria. These position-specific frequencies are converted into conditional probabilities by dividing them by the frequency with which the target word begins an $n$-gram which is provided in column 12 . The conditional probability that allows the word to be in any of the three subsequent positions was calculated with Equation 1. The 11th column gives the number of unique four-grams, rather than the summed frequencies associated with those four-grams. The analogous attributes are provided for the associate word.

The Word Entropy dataset includes 9,917 words as single lines of comma-delimited text in a single file. The first line of text is descriptive information. The attributes provided for each word are listed in the third column of Table 2. The second column contains the intermediate calculation described in Experiment 4. Subtracting this intermediate value from the logarithm of the total four-gram frequency of the word (column 12 for target words in the Predictive Links files) after dividing the intermediate value by the four-gram frequency of the word will produce the entropy value in column 3. In creating the Google dataset, Brants and Franz (2006) only included $n$-grams with a frequency higher than 40 entries. Our entropy calculation has treated the missing $n$ grams as negligible and excluded them as Brants and Franz (2006) did in the creation of the dataset. However, it would be possible to estimate the number of excluded 4-grams by making assumptions based on the target word's frequency as a single token. Access to this intermediate value allows future researchers to explore such assumptions. The last three attributes provide descriptive statistics for the four-gram frequencies associated with the word.

\section{References}

Balota, D. A., Pollatsek, A., \& Rayner, K. (1985). The interaction of contextual constraints and parafoveal visual information in reading. Cognitive Psychology, 17, 364-390.

Berger, A. L., Della Pietra, S. A., \& Della Pietra, V. J. (1996). A maximum entropy approach to natural language processing. Computational Linguistics, 22, 39-71.

Brants, T., \& Franz, A. (2006). Web 1T 5-gram, Version 1. Philadelphia: Linguistic Data Consortium, University of Pennsylvania.

Brown, P. F., Cocke, J., Della Pietra, S. A., Della Pietra, V. J., Jelinek, F., Lafferty, J. D., et al. (1990). A statistical approach to machine translation. Computational Linguistics, 16, 79-85.

Brown, P. F., Della Pietra, S. A., Della Pietra, V. J., Lai, J. C., \& Mercer, R. L. (1992). An estimate of an upper bound for the entropy of English. Computational Linguistics, 18, 31-40.

Bullinaria, J. A., \& Levy, J. P. (2007). Extracting semantic representations from word co-occurrence statistics: A computational study. Behavior Research Methods, 39, 510-526.

Carlberger, A., Carlberger, J., Magnuson, T., Palazuelos-Cagigas, S. E., Hunnicutt, M. S., \& Navarro, S. A. (1997, July). Profet, a new generation of word prediction: An evaluation study. Paper 
presented at the 2nd Workshop on NLP for Communication Aids, Madrid, Spain.

Chen, S. F., \& Goodman, J. (1998). An empirical study of smoothing techniques for language modeling (Tech. Rep. TR-10-98). Cambridge: Harvard University.

Chen, S. F., \& Rosenfeld, J. (1999). A Gaussian prior for smoothing maximum entropy models (Tech. Rep. CMU-CS-99-108). Pittsburgh: Carnegie Mellon University.

Collins, E. M., \& Loftus, E. F. (1975). A spreading activation theory of semantic processing. Psychological Review, 82, 407-428.

DeLong, K. A., Urbach, T. P., \& Kutas, M. (2005). Probabilistic word pre-activation during language comprehension inferred from electrical brain activity. Nature Neuroscience, 8, 11171121.

Dewey, G. (1929). Relative frequency of English speech sounds. Cambridge: Harvard University Press.

Ehrlich, S. F., \& Rayner, K. (1981). Contextual effects on word perception and eye movements during reading. Journal of Verbal Learning and Verbal Behavior, 20, 641-655.

Federmeier, K. D., \& Kutas, M. (1999). Right words and left words: Electrophysiological evidence for hemispheric differences in meaning processing. Cognitive Brain Research, 8, 373-392.

Federmeier, K. D., Wlotko, E. W., De Ochoa-Dewald, E., \& Kutas, M. (2007). Multiple effects of sentential constraint on word processing. Brain Research, 1146, 75-84.

Frank, A., \& Jaeger, T. F. (2008). Speaking rationally: Uniform information density as an optimal strategy for language production. In B. C. Love, K. McRae, \& V. M. Sloutsky (Eds.), Proceedings of the 30th Annual Conference of the Cognitive Science Society (pp. 939-944). Austin: Cognitive Science Society.

Frisson, S., Rayner, K., \& Pickering, M. J. (2005). Effects of contextual predictability and transitional probability on eye movements during reading. Journal of Experimental Psychology: Learning Memory and Cognition, 31, 862-877.

Genzel, D., \& Charniak, E. (2002). Entropy rate constancy in text. In Proceedings of the Association of Computational Linguistics (pp. 199-206). Stroudsburg, PA: Association for Computational Linguistics

Genzel, D., \& Charniak, E. (2003). Variation of entropy and parse trees of sentences as a function of the sentence number. In M. Collins \& M. Steedman (Eds.), Proceedings of the 2003 Conference on Empirical Methods in Natural Language Processing (pp. 65-72). Stroudsburg: Association for Computational Linguistics.

Good, I. J. (1953). The population frequencies of species and the estimation of population parameters. Biometrika, 40, 237-264.

Griffiths, T. L., Steyvers, M., \& Tenenbaum, J. B. (2007). Topics in semantic representation. Psychological Review, 114, 211-244.

Grignetti, M. (1964). A note on the entropy of words in printed English. Information Control, 7, 304-306.

Huettig, F., Quinlan, P. T., McDonald, S. A., \& Altmann, G. T. M. (2006). Models of high-dimensional semantic space predict language-mediated eye movements in the visual world. Acta Psychologica, 121, 65-80. doi:10.1016/j.actpsy.2005.06.002

Hunnicutt, S. (1985). Lexical prediction for a text-to-speech system. Speech, Music and Hearing: Quarterly Progress and Status Report, 26, 047-055.

Hutchison, K. A., Balota, D. A., Cortese, M. J., \& Watson, J. M. (2008). Predicting semantic priming at the item level. Quarterly Journal of Experimental Psychology, 61, 1030-1066. doi:10.1080/ 17470210701438111

Jaeger, T. F. (2010). Redundancy and reduction: Speakers manage information density. Cognitive Psychology, 61, 23-62.

Jelinek, F. (1976). Continuous speech recognition by statistical methods. Proceedings of the IEEE, 64, 532-556. doi:10.1109/ PROC.1976.10159
Keller, F. (2004). The entropy rate principle as a predictor of processing effort: An evaluation against eye-tracking data. In Proceedings of the Conference on Empirical Methods in Natural Language Processing (pp. 317-324). Stroudsburg, PA: Association for Computational Linguistics

Kučera, H., \& Francis, W. N. (1967). Computational analysis of presentday American English. Providence: Brown University Press.

Kutas, M., \& Federmeier, K. D. (2011). Thirty years and counting: Finding meaning in the N400 component of the event-related brain potential (ERP). Annual Review of Psychology, 62, 621647. doi:10.1146/annurev.psych.093008.131123

Kutas, M., \& Hillyard, S. A. (1984). Brain potentials during reading reflect word expectancy and semantic association. Nature, 307, $161-163$.

Landauer, T. K., \& Dumais, S. T. (1997). A solution to Plato's problem: The latent semantic analysis theory of acquisition, induction, and representation of knowledge. Psychological Review, 104, 211-240.

Levy, R., \& Jaeger, T. F. (2007). Speakers optimize information density through syntactic reduction. In B. Schlökopf, J. Platt, \& T. Hoffman (Eds.), Advances in neural information processing systems (NIPS) (Vol. 19, pp. 849-856). Cambridge: MIT Press.

Lund, K., \& Burgess, C. (1996). Producing high-dimensional semantic spaces from lexical co-occurrence. Behavior Research Methods, Instruments, \& Computers, 28, 203-208.

McDonald, S. (2000). Environmental determinants of lexical processing effort. Unpublished $\mathrm{PhD}$ dissertation, University of Edinburgh

McDonald, S., \& Lowe, W. (1998). Modeling functional priming and the associative boost. In Proceedings of the 20th Annual Conference of the Cognitive Science Society (pp. 667- 680). Mahwah, NJ: Erlbaum

McDonald, S. A., \& Shillcock, R. C. (2003). Eye movements reveal the on-line computation of lexical probabilities. Psychological Science, 14, 648-652.

McNamara, T. P. (2005). Semantic priming: Perspectives from memory and word recognition. New York: Psychology Press.

Navigli, R. (2009). Word sense disambiguation: A survey. $A C M$ Computing Surveys, 41, 1-69. doi:10.1145/1459352.1459355

Nelson, D. L., McEvoy, C. L., \& Dennis, S. (2000). What is free association and what does it measure? Memory \& Cognition, 28, 887-899.

Nelson, D. L., McEvoy, C. L., \& Schreiber, T. A. (2004). The University of South Florida free association, rhyme, and word fragment norms. Behavior Research Methods, Instruments, \& Computers, 36, 402-407.

Norris, D. (2006). The Bayesian Reader: Explaining word recognition as an optimal Bayesian decision process. Psychological Review, $113,327-357$.

Norris, D. (2009). Putting it all together: A unified account of word recognition and reaction-time distributions. Psychological Review, 116, 207-219.

Rayner, K., Ashby, J., Pollatsek, A., \& Reichle, E. D. (2004). The effects of frequency and predictability on eye fixations in reading: Implications for the E-Z Reader model. Journal of Experimental Psychology, 30, 720-732.

Rayner, K., \& Well, A. D. (1996). Effects of contextual constraint on eye movements in reading: A further examination. Psychonomic Bulletin \& Review, 3, 504-509.

Schustack, M. W., Ehrlich, S. F., \& Rayner, K. (1987). Local and global sources of contextual facilitation in reading. Journal of Memory and Language, 26, 322-340.

Shannon, C. E. (1948). A mathematical theory of communication. Bell Systems Technical Journal, 27, 379-423, 623-665. Retrieved from http://cm.bell-labs.com/cm/ms/what/shannonday/paper. html 
Shannon, C. E. (1951). Prediction and entropy of printed English. Bell Systems Technical Journal, 30, 50-64.

Sigurd, B., Eeg-Olofsson, M., \& van de Weijer, J. (2004). Word length, sentence length and frequency-Zipf revisited. Studia Linguistica, 58, 37-52.

Steyvers, M., \& Tenenbaum, J. B. (2005). The large-scale structure of semantic networks: Statistical analyses and a model of semantic growth. Cognitive Science, 29, 41-78.

Talbot, D., \& Osborne, M. (2007). Smoothed Bloom filter language models: Tera-scale LMs on the cheap. In E. Ringger (Ed.), Proceedings of the 2007 Joint Conference on Empirical Methods in Natural Language Processing and Computational Natural Language Learning (pp. 468-476). Stroudsburg: Association for Computational Linguistics.

Tversky, A., \& Kahneman, D. (1974). Judgment under uncertainty: Heuristics and biases. Science, 185, 1124-1131.

Van Berkum, J. J. A., Brown, C. M., Zwitserlood, P., Kooijman, V., \& Hagoort, P. (2005). Anticipating upcoming words in discourse: Evidence from ERPs and reading times. Journal of
Experimental Psychology: Learning, Memory, and Cognition, 31, 443-467.

Wandmacher, T., \& Antoine, J. Y. (2007). Methods to integrate a language model with semantic information for a word prediction component. In E. Ringger (Ed.), Proceedings of the 2007 Joint Conference on Empirical Methods in Natural Language Processing and Computational Natural Language Learning (pp. 506513). Stroudsburg: Association for Computational Linguistics.

Wicha, N. Y., Moreno, E. M., \& Kutas, M. (2004). Anticipating words and their gender: An event-related brain potential study of semantic integration, gender expectancy, and gender agreement in Spanish sentence reading. Journal of Cognitive Neuroscience, 16, 1272-1288.

Zhai, C., \& Lafferty, J. (2004). A study of smoothing methods for language models applied to information retrieval. ACM Transactions on Information Systems, 22, 179-214. doi:10.1145/ 984321.984322

Zipf, G. K. (1935). The psycho-biology of language. Cambridge: MIT Press. 